- Implications for undergraduate and postgraduate teaching.

- How can guidelines be better discriminated and implemented.

- Is there an under-prescription of dental radiography for children and what factors may CPD PAPER influence this?

\title{
An investigation into the use of bitewing radiography in children in Greater Glasgow
}

\author{
G. K. Taylor ${ }^{1}$ and L. M. D. Macpherson ${ }^{2}$
}

\begin{abstract}
Aim In recent years there have been a number of reviews and guidelines published with respect to the diagnosis and management of caries in children. Bitewing radiography remains the recommended method of choice for caries diagnosis in most circumstances. The aims of the study were to investigate the usage of bitewing radiography by general dental practitioners (GDPs) in Greater Glasgow, for the diagnosis of caries in children and to assess the usefulness of the technique as perceived by these dental practitioners.

Design A questionnaire for self completion was sent to all GDPs with an NHS list number in the Greater Glasgow area.

Results An 80\% response rate was obtained with 303 GDPs responding. Less than half the dentists (44\%) indicated that they always carried out caries risk assessments for all children, but 71\% reported doing this for 6 and 7 -year-old children. Approximately $60 \%$ of dentists stated they had read the recent radiation and caries management guidelines. Only $72 \%$ stated that they used radiography as a caries diagnostic tool in children, with 12 GDPs (4\%) indicating they would never consider using bitewing radiography in children. Only 17\% would consider taking bitewing radiographs in children under 6 years, and the majority of dentists (61\%) reported that they would first consider taking radiographs in the 6-11-year-old age group. However, only a low proportion of children in this age band had dental x-rays taken. Bitewing radiography was considered to be more important for 12-year-olds than for 6-year-olds. Recommended methods for decreasing radiation exposure such as rectangular collimation and film holders were not being used universally, with $41 \%$ and $58 \%$

respectively using these devices.

Conclusions A significant proportion of dentists in Greater Glasgow are not complying with recent guidelines and recommendations and it would appear that the value of bitewing radiography as a diagnostic tool in children is not being fully exploited.
\end{abstract}

${ }^{1}$ GDP, Clinical Governance Adviser Greater Glasgow Primary Care NHS Trust and Lead Dental Audit Facilitator Argyll and Clyde NHS Board; ${ }^{2 *}$ Senior Lecturer and Honorary Consultant in Dental Public Health, University of Glasgow Dental School, 378 Sauchiehall Street, Glasgow, G2 3JZ

Correspondence to: Lorna M. D. Macpherson

*Email: I.macpherson@dental.gla.ac.uk

\section{Refereed paper}

Received 18.02.03; Accepted 30.06.03

doi:10.1038/sj.bdj.4811228

๑ British Dental Journal 2004; 196: 563-568
Dental caries in children remains a significant problem and is a priority to the NHS in Scotland. In recent years there have been a number of reviews and guidance documents published in the UK with respect to the diagnosis and management of this disease in children. ${ }^{1-3}$

Guidance published by the Faculty of General Dental Practitioners (UK) in $1998^{1}$ covered all aspects of radiography in primary dental care. That document, in common with much of the literature, agreed that radiographs were an essential adjunct to a clinical examination and should be used for both operative and preventive dentistry. It stated that categorisation of patients, by means of an assessment of caries risk, was the most effective way to determine the appropriate frequency of exposure to radiographs.

At approximately the same time, a recommendation was made that bitewing radiographs should be taken as soon as the co-operation of the child allowed, but particularly in the pre-school age group for the high-risk child. ${ }^{2}$ It was anticipated that for children and adolescents, bitewings should be taken at two-yearly intervals, but for those with evidence of caries on previous films, this may be increased to as frequently as every six months.

SIGN Guideline No. 47 Preventing Dental Caries in Children at High Caries Risk, ${ }^{3}$ was sent to all general dental practitioners in Scotland in 2000. It was designed to target caries-prevention in children's permanent teeth and covered the $6-16$-year-old age range. It, too, recommended the use of a caries-risk assessment as the basis for diagnostic, preventive and operative care, and reiterated that bitewing radiographs were essential as an adjunct to the patient's first clinical examination.

Many articles have been written on the size of the increase in yield that bitewing radiographs give when detecting approximal caries relative to a clinical examination alone. In 1990, Kidd and Pitts published an article reviewing the results of 29 studies in this field. ${ }^{4}$ The amount of undetected caries varied, but the findings showed that the use of bitewing radiographs was essential if much approximal caries was not to be missed. It was considered particularly important for the detection of early lesions, giving the potential for preventive rather than operative care.

With regard to occlusal caries, a report published in 1990 involving adolescents in Scotland, showed that a large proportion of occlusal surfaces that were diagnosed as clinically sound 
had caries affecting dentine when examined using bitewing radiography as an adjunct. ${ }^{5}$ It was concluded that, even with a trained clinical examiner and optimum conditions, hidden caries was a major problem when no radiographs were exposed.

While there is some risk to patients and staff when using ionising radiation, this risk can be reduced through proper training, use of appropriate films and radiographic equipment, and continued professional development to ensure radiation protocols are up-to-date and being implemented efficiently. ${ }^{6}$ Thus, in recent years a number of guidelines and recommendations have been published in parallel with new legislation on radiation. ${ }^{7-9}$ The major issues they highlight are safety for patients and staff, and the timing of and selection criteria for taking radiographs.

Much guidance is therefore available for dentists with respect to caries-risk assessment and the diagnosis and treatment of caries in children. Since the publication of the recent guidelines, there have been no surveys of Scottish dentists' attitudes and practices in relation to these issues.

The main aims of the study were, therefore, to explore the usage and perceived usefulness of caries-risk assessments and dental radiography in children within primary dental care in Greater Glasgow.

The objectives were:

1. To determine if dentists are carrying out a caries risk assessment for child patients categorising them into high, medium and low risk.

2. To investigate the methods being used by dentists for the diagnosis of caries in children.

3. To determine the types of $\mathrm{x}$-ray equipment dentists have access to.

4. To examine the perceived usefulness of dental radiography in children.

5. To investigate whether the recommendations contained in recent guidelines concerning the use by dentists of bitewing radiography in children are being followed.

\section{METHODS}

A postal self-completion questionnaire was developed and sent to all GDPs with an NHS list number in Greater Glasgow.

Study questions were chosen that would provide data relating to: i) Socio-demographic information about respondents' dental practice; ii) Conduct of caries risk assessments; iii) Access to and use of radiographic equipment; iv) Practitioner's views on the use of radiography in children and v) Current practice regarding x-ray use in children.

The questionnaire used was based, in part, upon that used in a previous study by Pitts and Fyffe. ${ }^{10}$ The attitudinal questions contained statements to which the dentists were asked to indicate the level of perceived importance on a four point scale ranging from 1 = unimportant to $4=$ very important.

Initial piloting was carried out in Argyll and Clyde, a neighbouring health board area. It was estimated that the questionnaire would take between 5 and 10 minutes to complete.

For the main study, a list of general dental practitioners with an NHS list number was obtained from Greater Glasgow Primary Care NHS Trust. The questionnaire was sent to 384 practitioners in late March 2002. Dentists were requested to complete and return the questionnaires within a three-week period. A follow-up letter was compiled and the questionnaires were sent again to the nonrespondents, four weeks after the original questionnaire.

The data were entered into an Excel database and subsequently analysed using Minitab (version 13). Analysis involved descriptive statistics and cross tabulation, with potential associations tested for statistical significance using Pearson's chi-squared tests.

\section{RESULTS}

\section{General and demographic data}

A total of 303 questionnaires were returned. This represented a response rate of $80 \%$. Overall, $69 \%$ of the respondents were male and 31\% were female. Regarding years since graduation, 40\% had been qualified for between 11 and 20 years, while the proportions who had been qualified for up to 10 years, 21-30 years and more than 30 years were $21 \%, 28 \%$ and $11 \%$, respectively. Forty dentists $(13 \%)$ were in practices with vocational trainees, with 16 being formal trainers.

With regard to socio-economic status of practice location, 51\% of the GDPs practised in DEPCAT 6 and 7 areas, with only 22\% working in a DEPCAT 1, 2 or 3 location. The remaining $27 \%$ had practices located in DEPCAT 4 or 5 areas.

\section{Case-mix}

Over half of the respondents (58\%) indicated they saw all their adult patients on the NHS, and 285 (94\%) saw all children on the NHS. Only seven dentists (2\%) reported seeing all their adults privately. All saw some children on the NHS, with only five (2\%) seeing more than $10 \%$ of children privately.

\section{Frequency with which caries risk assessments are carried out}

The dentists were asked if they carried out a caries risk assessment, and 134 (44\%) stated they 'always' carried out such an assessment for children. Almost one third (29\%) indicated that they never carried out this procedure. Of the $27 \%$ 'sometimes' performing an assessment, $8 \%$ of the total restricted this only to 6 and 7 -yearolds.

Factors that were perceived to influence categorisation of individual patients into high, medium or low caries-risk groups are shown in Table 1. Past caries experience and level of oral hygiene were considered to be relevant factors by $70 \%$ and $63 \%$ of dentists, respectively.

\begin{tabular}{|c|c|}
\hline Factor & $\begin{array}{l}\text { Percentage of respondents } \\
\text { influenced by factor }(\%)\end{array}$ \\
\hline Age of patient & $114(38 \%)$ \\
\hline Past caries experience & $213(70 \%)$ \\
\hline Caries in other family members & $141(46 \%)$ \\
\hline Regularity of attendance & $128(42 \%)$ \\
\hline Diet & 167 (55\%) \\
\hline Oral hygiene & 191 (63\%) \\
\hline
\end{tabular}

\section{Methods of caries diagnosis}

While all practitioners used direct vision as a method of caries diagnosis, only $72 \%$ stated that they used radiography as a caries diagnostic tool in children. Tooth separation was reported to be used by $11(4 \%)$ respondents.

All GDPs had access to intra-oral radiography, and around one quarter of GDPs (26\%) reported having access to panoramic radiography. Only a small number of dentists $(25,8 \%)$ reported use of digital radiography. Six of those also used conventional radiography.

The majority (83\%) of GDPs indicated use of a long cone technique, with the remainder using a short cone. Half the respondents indicated they never used rectangular collimation, while the percentages stating they always or sometimes used this technique were $42 \%$ and $8 \%$, respectively. Forty seven per cent of dentists who had been qualified for less than 20 years used rectangular collimation, compared with $26 \%$ of dentists 
who had been qualified more than 30 years $(P$ value $<0.001)$. Just over half of GDPs (52\%) in totally NHS practices use rectangular collimation, compared with $44 \%$ in mixed practices. A high proportion $(78 \%)$ of dentists in vocational training practices indicated they always used rectangular collimation. Dentists who stated they always carried out a caries-risk assessment were more likely to indicate use of rectangular collimation $(P=0.04)$.

\section{Use of film holders}

The proportions of dentists using film holders or paper tabs to hold the film in the correct position were $58 \%$ and $42 \%$, respectively. A significant negative association between number of years qualified and use of film holders was found $(P<0.001)$, with the percentage of those using film holders decreasing with the number of years qualified.

\section{Factors influencing the decision of whether or not to take bitewing radiographs of a child}

The dentists were asked to rank on a scale of 1 (unimportant) to 4 (very important) various factors that may influence their decision to take bitewing radiographs. The results are shown in Table 2. Patient 'co-operation' and 'past caries experience' were considered to be very important factors by $42 \%$ and $52 \%$ of the respondents, respectively.

\section{Age at which dentists would first consider taking bitewing radiographs of children}

Only 12 GDPs (4\%) reported they would consider taking bitewing radiographs in children under the age of 3 years, with 13\% indicating between 3 and 5 years of age. The majority of GDPs (61\%) would first consider taking bitewings of a child between the ages of 6 and 11 years, with 18\% between 12 and 17 years, while 4\% stated that they would never consider the use of radiography in children.

\section{Recall interval for taking bitewing radiographs}

A high proportion of respondents (65\%) reported that for child patients, the interval between bitewing radiography depended on the caries rate of the child, while $12 \%$ of GDPs stated that they carried out an annual radiographic examination.

\section{Percentage of children having bitewing radiographs taken}

The dentists were asked to estimate what percentage of 6 and 12year-old children that attended them for routine care, would have had bitewing radiographs taken. The results, shown in Table 3 indicate that only a quarter of GDPs estimate that more than 1 in 5 of their 6-year-old patients would have had a bitewing radiograph taken, with the corresponding percentage for 12-year-olds being $48 \%$. Only $40 \%$ of the dentists qualified for over 30 years had taken any bitewings of 6-year-old children, compared with 84\% of those dentists qualified less than 10 years $(P<0.001)$, a similar pattern was seen in relation to 12 -year-olds.

\section{Guidelines}

Sixty four per cent of respondents stated they had read the SIGN Guideline relating to preventing caries in high caries-risk children. Dentists who had read the SIGN Guideline were more likely to always carry out a caries risk assessment, compared with those who had not $(P=0.001)$.

The points relevant to dentistry from radiation regulations IRR99 and IR(ME)R2000 were summarised in the form of guidance notes and had been circulated to all primary care dentists in 2001. Sixty per cent of dentists stated that they had read the regulations. Those who stated that they had read the SIGN Guideline were more likely to have indicated that they had also read the radiation regulations $(P<0.001)$.

Forty one per cent and 31\% of those who had read the SIGN Guidelines and radiation regulations, respectively stated that the documents had influenced their practice with regard to number of radiographs taken.

\section{Audit of radiographs}

Approximately half (52\%) of the dentists stated that they audited the quality of their radiographs. Sixty four per cent (116) of those indicating that they had read the radiation regulations said they routinely audit the quality of their radiographs, compared with 65 dentists (36\%) who had not read the regulations $(P<0.001)$.

\section{Perceived usefulness of bitewing radiographs in 6-year-old and 12-year-old children}

The dentists were asked questions concerning the perceived usefulness of bitewing radiographs in 6-year-old and 12-year-old children. The results are shown in Tables 4 and 5, respectively.

\begin{tabular}{|c|c|c|c|c|c|}
\hline & & \multicolumn{4}{|c|}{ Level of importance* } \\
\hline & & $\begin{array}{c}1 \\
\text { №. }(\%)\end{array}$ & $\begin{array}{c}2 \\
\text { No. }(\%)\end{array}$ & $\begin{array}{c}3 \\
\text { No. }(\%)\end{array}$ & $\begin{array}{c}4 \\
\text { No. }(\%)\end{array}$ \\
\hline Child co-operation & $n=299$ & $14(5 \%)$ & $54(18 \%)$ & 106 (35\%) & $125(42 \%)$ \\
\hline Past caries & $n=299$ & $14(5 \%)$ & $20(7 \%)$ & $110(37 \%)$ & $155(52 \%)$ \\
\hline Caries in siblings & $n=298$ & $40(13 \%)$ & $100(33 \%)$ & $116(39 \%)$ & $42(14 \%)$ \\
\hline Oral hygiene & $n=299$ & $14(5 \%)$ & $54(18 \%)$ & $126(42 \%)$ & $105(35 \%)$ \\
\hline Radiation risk & $n=299$ & $99(33 \%)$ & $91(30 \%)$ & $55(18 \%)$ & $54(18 \%)$ \\
\hline Remuneration & $n=299$ & $98(33 \%)$ & $90(30 \%)$ & $52(17 \%)$ & $59(20 \%)$ \\
\hline Time available & $n=298$ & $78(26 \%)$ & $93(31 \%)$ & $94(32 \%)$ & $33(11 \%)$ \\
\hline
\end{tabular}

\begin{tabular}{|c|c|c|c|c|c|}
\hline & & \multicolumn{4}{|c|}{ Percentage children (\%) } \\
\hline & & $0 \%$ & $1-20 \%$ & $21-40 \%$ & $41+\%$ \\
\hline 6 year-olds & $N=302$ & $85(28 \%)$ & $143(47 \%)$ & $35(12 \%)$ & $39(13 \%)$ \\
\hline 12year-olds & $N=301$ & $39(13 \%)$ & $118(39 \%)$ & $65(22 \%)$ & $79(26 \%)$ \\
\hline
\end{tabular}


Table 4 Perceived usefulness of bitewings radiographs with regard to deciduous teeth of 6-year-olds

\begin{tabular}{|c|c|c|c|c|c|}
\hline & & $1^{*}$ & 2 & 3 & 4 \\
\hline Preventive care & $n=292$ & $43(15 \%)$ & $78(27 \%)$ & $102(35 \%)$ & $69(24 \%)$ \\
\hline Restoration approximal dentinal lesions & $n=292$ & $34(12 \%)$ & $102(35 \%)$ & $100(34 \%)$ & $56(19 \%)$ \\
\hline Occlusal caries & $n=288$ & $26(9 \%)$ & $49(17 \%)$ & $117(41 \%)$ & $96(33 \%)$ \\
\hline Assessing caries progression & $n=289$ & $27(9 \%)$ & $107(37 \%)$ & $95(33 \%)$ & $60(21 \%)$ \\
\hline
\end{tabular}

${ }^{*} 1$ = very valuable $; 4$ = little value

Table 5 Perceived usefulness of bitewings radiographs with regard to 12-year-olds

\begin{tabular}{|c|c|c|c|c|c|}
\hline & & $1^{*}$ & 2 & 3 & 4 \\
\hline Preventive care & $n=298$ & $141(47 \%)$ & $106(36 \%)$ & $30(10 \%)$ & $21(7 \%)$ \\
\hline Restoration approximal dentinal lesions & $n=296$ & $154(52 \%)$ & $105(35 \%)$ & $20(7 \%)$ & $17(6 \%)$ \\
\hline Occlusal caries & $n=296$ & $75(25 \%)$ & $129(44 \%)$ & $67(23 \%)$ & $25(8 \%)$ \\
\hline Assessing caries progression & $n=296$ & $85(29 \%)$ & $139(47 \%)$ & $51(17 \%)$ & $21(7 \%)$ \\
\hline
\end{tabular}

*1 = very valuable ; 4 = little value

\section{Six-year-olds}

Only 15\% of GDPs considered bitewing radiographs very valuable for diagnosing deciduous caries in 6-year-old children to allow lesions to be treated in a preventive manner. Similarly, only $12 \%$ of respondents perceived bitewing radiographs to be very valuable in allowing restorative treatment of approximally detected dentinal caries in deciduous teeth. The percentages considering bitewing radiographs to be very valuable for assessing caries progression and for detecting occlusal caries in the deciduous dentition were both $9 \%$

An additional question was asked relating to the perceived usefulness of bitewing radiography in detecting caries in the first permanent molars of 6-year-olds. Seventy-seven dentists (27\%) indicated that they considered bitewings to be very valuable, while 56 (19\%) perceived the technique to be of little value in this regard.

\section{2-year-olds}

Forty seven per cent of respondents considered bitewing radiographs very valuable for diagnosing caries in the teeth of 12year-old children so that detected lesions could be treated in a preventive manner, and 52\% perceived the technique to be very useful for permitting the restoration of approximally detected dentinal caries. A smaller percentage of dentists (25\%) thought bitewings very valuable for the detection of occlusal caries.

\section{Item of service payment for bitewing radiographs in children}

Almost all (92\%) GDPs thought there should be an item of service payment for bitewing radiography in children. If there was an item of service payment for taking bitewing radiographs, 137 GDPs $(46 \%)$ stated that the frequency with which they would take them would increase while 163 (54\%) said the frequency would not change. A lower proportion (40\%) of GDPs who had read the radiation regulations stated that their frequency would increase compared with those who had not read them (54\%) $(P=0.019)$.

\section{DISCUSSION}

The study obtained a relatively high response rate of $80 \%$ and the socio-demographic profile of respondents' practices is very similar to that of Greater Glasgow practitioners as a whole.

Within the general dental service in Scotland, an enhanced capitation payment was introduced in 1998, based on the deprivation score (DEPCAT) of the practice address. ${ }^{11}$ For 3-5-year-olds, an enhanced fee of $25 \%$ is paid for those registered with practices in
DECPAT 6 and 7 locations. More recently, in December 2001, the 'Caries Prevention Scheme' commenced. This gives a higher capitation payment for all registered 6 and 7-year-olds. ${ }^{12}$ At present, this represents increases of $£ 1.10$ and $£ 1.48$ per month for DEPCAT 1 to 3 and 4 to 7 locations, respectively. Dentists in the GDS must carry out 'an assessment of caries risk, including radiographs as required, to be noted in the patient record'. Guidance relating to appropriate radiographic use is contained within SIGN Guideline $47 .^{3}$ If caries risk is high, the GDPs must fissure seal the occlusal surfaces of the remaining first permanent molars, in-so-far as patient co-operation allows, in addition to delivering a preventive programme with dietary and fluoride advice. Should all four first permanent molars be missing or unsuitable for fissure sealing, an intensive preventive programme, including the use of topical fluoride varnish application should be instituted. Information to dentists relating to this requirement was circulated in November 2001, in the form of a letter from the Scottish Executive. The present study found that, four months later, approximately 30\% of GDPs stated they never carried out a caries risk assessment for any age group, with only $71 \%$ reporting to do this for 6 and 7-year-olds. Less than half of the dentists carried out a caries risk assessment for all age groups of children although its use has been recommended in the SIGN guideline. ${ }^{3}$ However, whether in some cases caries risk assessment forms part of routine practice without the practitioner formally recognising the term and recording the categorisation in the patient record is unknown and perhaps requires further investigation.

The numbers of dentists using digital radiography in the UK are reported to be on the increase. ${ }^{13}$ However, only a relatively small proportion of Greater Glasgow's dentists (8\%) used this technique. Digital systems for intra-oral radiography use two different methods of image capture. Storage phosphor plates (SPP) are similar in size to conventional dental $\mathrm{X}$-ray film, while solid-state detectors are bulkier and attached to cables. A recent study, ${ }^{14}$ involving Dutch GDPs, reported they found conventional film easier to handle pre-exposure, but digital sensors more user-friendly post-exposure. Of those working with digital radiography, two thirds used a SPP system. This was associated with fewer difficulties than solidstate systems with regard to receptor positioning. In a follow-up study, ${ }^{15}$ the authors reported that GDPs with digital radiographic systems were more inclined to take radiographs than those using conventional radiographs, and thus the dose-reduction associated with converting from conventional to digital may be lower than anticipated due to an increase in number of radiographs taken. 
In the past, short cones were used on the tube heads of x-ray machines. However, it is now known that they give a much higher skin dose than long cones, and they are unable to accept collimation/film holders. ${ }^{16}$ Consequently, the use of short cones is not recommended in the recent IR(ME)R2000 regulations. However, in the present study, $17 \%$ of the general dental practitioners indicated continued use of this technique.

The IR(ME)R2000 regulations state that the dentist must do everything possible to decrease the radiation dose. One way that this might be achieved is through the use of rectangular collimation. ${ }^{16}$ This current study showed that only $42 \%$ of GDPs always used collimation when taking radiographs. Furthermore, half the dentists reported that they never used collimation. The findings suggest that some dentists are either ignoring guidance, are unaware that the guidance exists, or are unaware of this technique. An additional factor could be the fact that greater precision is required when using rectangular collimation. Although vocational training practices are inspected to ensure they use collimation, only $78 \%$ of dentists involved in vocational training reported its use.

Film holders act as positioning devices, keeping the film at right angles to the X-ray beam and thereby aiding the production of high quality images. Their use is recommended in the IR(ME)R2000 regulations. The study showed that only slightly over half the study respondents used film holders. There were significant relationships between use of film holders and both the number of years qualified and the number of courses that the dentists had attended. This finding suggests that specific groups of practitioners, who have had no recent structured training in radiological techniques and radiological safety would benefit from training associated with radiological standards and practices.

It has been suggested that an average dental practitioner would be expected to devote at least 5\% (12.5 hours) of their 5 yearly 250-hour continuing professional development recertification cycle to radiology and radiation protection issues and that formal courses should be attended. ${ }^{9}$ The findings of this study indicate the importance of providing sufficient courses for practitioners to attend.

\section{The decision to take a radiograph}

Approximately half (52\%) of the respondents rated an individual's past caries experience as a very important factor to be considered in deciding whether or not to take a radiograph. Co-operation was enquired about because Pitts and co-workers showed in 1991 that many dentists lacked the confidence to take bitewing radiographs in children, as they assumed the patients would not tolerate the positioning devices. ${ }^{17}$ The current study found that $42 \%$ of the practitioners considered patient co-operation to be a very important factor influencing their decision of whether or not to take a radiograph.

Only a relatively low proportion of practitioners indicated that they considered time and remuneration to be important factors which influence their decision-making. This result is somewhat surprising considering the literature which exists relating to the perceived under funding of children's dentistry. ${ }^{18}$ Despite respondents indicating that remuneration was not an important issue with respect to the decision to take bitewing radiographs, in a later section of the questionnaire, 92\% of GDPs thought there should be an item of service payment for bitewing radiographs in children. If an item of service payment did exist, 46\% of the GDPs stated that the frequency with which they took such radiographs would increase. This suggests that payment may indeed be a secondary issue. In 1996, Rushton and co-workers, in a survey of GDPs in England that involved use of clinical scenarios, found there was a tendency towards under-prescription of bitewing radiographs for high-risk mixed-dentition patients and over-prescription for high- risk adults. ${ }^{19}$ They speculated on whether the remuneration system could have influenced the findings. If an item of service fee were to be introduced for radiography in children, there could be the potential for both positive and negative effects, and changes in practice would require monitoring to ensure appropriate use was being made of this diagnostic tool.

\section{Age of patient at which dentist would first consider taking radiographs}

Although it has been recommended that bitewing radiographs should be taken for high risk children in the pre-school age group, ${ }^{2}$ the present study found that only a very low proportion of dentists (17\%) considered use of such radiographs in children aged five years or under. These findings raise important issues given the fact that by three years of age, over $60 \%$ of children from deprived areas of Glasgow have experienced dental disease, and that 54\% of Glasgow's 5-year-olds have unrestored dentinal decay. ${ }^{20}$ Reasons for the low usage of radiography may include lack of knowledge, safety concerns, co-operation worries, remuneration factors or uncertainty regarding the benefits of restorative treatment of caries in the deciduous dentition. ${ }^{21,22}$ While an increase in bitewing radiography could be advantageous in detecting lesions at an earlier stage, if preventive or restorative care is not going to result from such findings, the benefit of radiography among this population group has to be questioned. Thus, further work is required to investigate in more detail the reasons for the low radiographic usage and low Care Index associated with high caries-risk children in Glasgow.

The recent SIGN guideline, relating to 6-16 year-olds, recommended that radiographs should form part of an initial assessment, with the frequency thereafter being dependent on the caries-risk status of the child. However, the present study found that almost $30 \%$ of practitioners stated that they did not use radiography as a caries diagnostic tool in children. Furthermore, it was estimated that only a relatively small proportion of child patients, attending for routine care, would have had bitewing radiographs taken by the age of 12 years.

\section{Dentists reading guidelines and regulations}

At the time of the study (one year and three months after distribution of SIGN Guideline No. 47), only 64\% of GDPs stated that they had read the document. Similarly, just under two thirds of the dentists had read the Guidance Notes for Dental Practitioners on the Safe Use of X-Ray Equipment, ${ }^{9}$ nine months after distribution to all primary care dentists in Scotland. The fact that a dentist has read a guideline does not necessarily mean that they have understood or implemented it. However, the study found that those who stated that they had read the guidelines were more likely to indicate that they followed practices recommended in the documents eg caries risk assessment and use of collimation. Thus, the relationship between reading guidelines and the implementation of their recommendations requires further investigation. Consequently, primary care trusts can have an important role to play in raising the awareness of such documents and providing education to facilitate their implementation. Similarly, Rushton and coworkers highlighted the need for greater emphasis to be placed on radiographic selection criteria in dental education. ${ }^{23}$

\section{Audit of radiographs}

IR(ME)R $2000^{8}$ states that all dentists should audit the quality of their radiographs. However, in the study, only approximately half the dentists stated that they undertook this activity. The majority of dentists (62\%) who were involved in vocational training indicated they had carried out a radiograph audit. However, this figure is still low, considering that the practitioners will all have been asked specifically about this activity during their practice inspec- 
tion. Reasons for dentists not auditing the quality of their radiographs may include time factors, their lack of knowledge of audit, or a perceived view that audit is a burden. The new Dental practice advisor checklist, introduced in 2001, now has a question relating to radiographic audit. ${ }^{24}$ This, in conjunction with additional GDS funding for audit, ${ }^{25}$ may result in an increased compliance rate.

\section{Perceived usefulness of bitewing radiographs in 6-year-old and 12-year-old children}

In a previous study, ${ }^{10}$ dentists were asked about the usefulness of bitewing radiography for detecting approximal caries, and 73\% of respondents considered it to be very valuable. In the wake of increased understanding of the dynamics of the caries process over the past two decades, ${ }^{26}$ practitioners were asked about the role of radiographs for the diagnosis of caries for preventive as well as restorative treatment. The results showed that a similar number of dentists found bitewings very valuable for both purposes. The results indicate that a higher proportion of Glasgow dentists consider bitewing radiographs to be 'very valuable' for approximal caries diagnosis in older children than in younger children. This perhaps relates to the greater importance given to permanent teeth. However, even for 12-year-olds, the figures were lower than reported in $1987 .{ }^{10}$ The reasons for this finding require further exploration. While radiation risk may be one factor, Kidd and Pitts ${ }^{4}$ have stated that concern to minimise the number of radiographic exposures must be carefully balanced against the ethical difficulties of failing to use a technique which has been shown to detect lesions hidden from a clinical examination.

It is recognised that the diagnosis of occlusal caries is challenging and that limitations exist concerning conventional methods of lesion detection. ${ }^{27}$ Much work is therefore underway at present to develop new diagnostic tools, with greater sensitivity and reproducibility. Problems with current methods, including bitewing radiography, may explain in part, why a lower proportion of practitioners considered this diagnostic tool to be very important for the diagnosis of occlusal as compared with approximal caries.

As it is known that the behaviour of lesions will vary depending on localised conditions, ${ }^{26}$ it was expected that the proportion of dentists perceiving bitewings to be very valuable for assessing caries progression would have been higher than in 1987. However, the percentage was actually lower. Whether this could be due to the perceived rapid rate of progression of lesions amongst this high-risk population or to patterns of child dental attendance in Glasgow is unknown.

\section{CONCLUSIONS}

The study found that less than half the dentists in Glasgow stated that they always carried out a caries risk assessment for their child patients, even although the procedure was recommended in a SIGN guideline in 2000. Similarly, in relation to radiological safety issues, it would appear that some dentists are not complying with recent guidelines and regulations. Thus, the study raises important issues associated with the dissemination and implementation of guidelines and regulations which require to be addressed.
It is recognised that the potential risks and benefits of ionising radiation must be considered when contemplating the use of dental radiography and that use of caries-risk assessment procedures can help with the decision-making process. However, this study found that the proportion of children having bitewing radiographs was very low in a high caries-risk area, thus raising questions concerning the current management of child patients with early caries lesions. Further research is required to explore these issues further.

The authors are grateful to the general dental practitioners who participated in this study.

1. Faculty of General Dental Practitioners (UK) Working Party. Selection criteria for dental radiography. Faculty of General Dental Practitioners (UK) The Royal College of Surgeons of England, 1998.

2. Nunn J, Crawford P, Page J, Winter G. British Society of Paediatric Dentistry: A policy document on the dental needs of children. Int J Paediat Dent 1997; 7: 203-207.

3. Scottish Intercollegiate Guideline Network 47. Preventing caries in children at high risk. Edinburgh, 2000.

4. Kidd E A M, Pitts N B. A reappraisal of the value of the bitewing radiograph in the diagnosis of posterior approximal caries. Br DentJ 1990: 169: 195-200.

5. Creanor S L, Russell J I, Strang D M, Stephen K W, Burchell C K. The prevalence of clinically undetected occlusal dentine caries in Scottish adolescents. Br Dent J 1990; 169: $126-129$

6. Council on Dental Materials, Instruments and Equipment. Recommendations in radiographic practices: an update, 1988. J Am Dent Assoc 1989; 118: 115-117.

7. The lonising Radiations Regulations 1999. SI 1999 No 3232. London: The Stationery Office.

8. The lonising Radiation (Medical Exposure) Regulations 2000.SI 2000 No 1059. London: The Stationery Office.

9. National Radiological Protection Board. Guidance notes for dental practitioners on the safe use of $x$-ray equipment. Department of Health, 2001.

10. Pitts N B, Fyffe H E. Scottish dentists' use of and opinions regarding bitewing radiography. Dentomaxillofac Radio/ 1991; 20: 214-218.

11. Memorandum to NHS: 1998 PCA(D)9. Edinburgh: Scottish Office, 1998

12. Scottish Dental Practice Division. Amendment 81 to Statement of Dental Remuneration. November 2001.

13. Brown J E. Advances in dental imaging. Prim Dent Care 2001; 8: 59-62.

14. Berkhout W E R, Sanderink G C H, Van der Stelt P F. A comparison of digital and film radiography in Dutch dental practices assessed by questionnaire. Dentomaxillofac Radio/ 2002; 31: 93-99.

15. Berkhout W E R, Sanderink G C H, Van der Stelt P F. Does digital radiography increase the number of intraoral radiographs? A questionnaire study of Dutch dental practices. Dentomaxillofac Radio/ 2003; 32: 124-127.

16. Horner K, Hirschmann P N. Dose reduction in dental radiography. J Dent 1990; 18: $171-184$.

17. Pitts N B, Hamood S S, Longbottom C, Rimmer PA. The use of bitewing positioning devices in children's dentistry. Dentomaxillofac Radio/ 1991; 20: 121-126.

18. Curzon M E J, Pollard M A. Do we still care about children's teeth? BrDent J 1997; 182: $242-244$.

19. Rushton VE, Horner $\mathrm{K}$, Worthington HV. A two-centre study to determine dentists' agreement with current guidelines on the frequency of bitewing radiography. Commun Dent Oral Epidemiol 1996; 24: 175-181.

20. Scottish Health Boards' Dental Epidemiological Programme. The dental health of 5 year olds in Greater Glasgow 2001/2002. University of Dundee, 2002.

21. Milsom K M, Tickle $M, B$ linkhorn A S. Dental pain and dental treatment of young children attending the general dental service. Br Dent J 2002; 192: 280-284.

22. Levine R S, Pitts N B, Nugent $Z$ J. The fate of 1,587 unrestored carious deciduous teeth: a retrospective general dental practice based study from northern England. $\mathrm{Br}$ Dent J2002; 193: 99-103.

23. Rushton VE, Horner $K$, Worthington $H V$. Factors influencing the frequency of bitewing radiography in general dental practice. Commun Dent Oral Epidemiol 1996; 24: $272-276$

24. Dental practice inspection checklist. Greater Glasgow Primary Care Trust, 2001.

25. Scottish Dental Practice Division. Statement of Dental Remuneration. April 2002.

26. Pitts N B, Kidd E A M. Some of the factors to be considered in the prescription and timing of bitewing radiography in the diagnosis and management of dental caries. J Dent 1992; 20: 74-84.

27. McComb D, Tam L E. Diagnosis of occlusal caries: Part I. Conventional methods. J Can DentAssoc 2001; 67: 454-457. 